\title{
Heat kernel estimates for a class of higher order operators on Lie groups
}

by

\author{
Nick Dungey (Sydney)
}

\begin{abstract}
Let $G$ be a Lie group of polynomial volume growth. Consider a differential operator $H$ of order $2 m$ on $G$ which is a sum of even powers of a generating list $A_{1}, \ldots, A_{d^{\prime}}$ of right invariant vector fields. When $G$ is solvable, we obtain an algebraic condition on the list $A_{1}, \ldots, A_{d^{\prime}}$ which is sufficient to ensure that the semigroup kernel of $H$ satisfies global Gaussian estimates for all times. For $G$ not necessarily solvable, we state an analytic condition on the list which is necessary and sufficient for global Gaussian estimates. Our results extend previously known results for nilpotent groups.
\end{abstract}

1. Introduction. Let $G$ be a non-compact, connected, unimodular Lie group with Lie algebra $\mathfrak{g}$, and assume that $G$ has polynomial volume growth. Consider a differential operator

$$
H=H^{(m)}=(-1)^{m} \sum_{i=1}^{d^{\prime}} A_{i}^{2 m},
$$

where $m$ is a positive integer and $A_{1}, \ldots, A_{d^{\prime}}$ are right invariant vector fields on $G$ corresponding to a list of generators $a_{1}, \ldots, a_{d^{\prime}} \in \mathfrak{g}$ of the Lie algebra. It is known that $H$ generates a semigroup $S_{t}=e^{-t H}, t>0$, in the spaces $L^{p}=L^{p}(G ; d g), 1 \leq p \leq \infty$, where $d g$ denotes a fixed Haar measure on $G$. Moreover, $S_{t}$ acts via a convolution $S_{t} f=K_{t} * f, f \in L^{p}, t>0$, where $K_{t}: G \rightarrow \mathbb{R}$ is a smooth function satisfying the following "local" Gaussian bounds: for each $t_{0} \in(0, \infty)$, there exist $c, b>0$ such that

$$
\left|K_{t}(g)\right| \leq c t^{-D^{\prime} /(2 m)} e^{-b\left(\varrho_{A}(g)^{2 m} / t\right)^{1 /(2 m-1)}}
$$

for all $t \in\left(0, t_{0}\right]$ and $g \in G$ (see [16] and [11]). Here $\varrho_{A}: G \rightarrow[0, \infty)$ is the standard Carathéodory modulus associated with $A_{1}, \ldots, A_{d^{\prime}}$, and $D^{\prime} \in \mathbb{N}$ is a local dimension associated with $\varrho_{A}$ (for background, see $[19,18]$ ).

Since $G$ has polynomial growth, there is a $D \in \mathbb{N}$ such that an estimate $c^{-1} r^{D} \leq d g(B(r)) \leq c r^{D}$ holds for all $r \geq 1$, where $B(r):=\{g \in G$ : $\left.\varrho_{A}(g)<r\right\}$ is the ball of radius $r$ associated with $\varrho_{A}$. Let us say that $H$

2000 Mathematics Subject Classification: 22E30, 22E25, 35B40. 
satisfies global Gaussian bounds if there exist $c, b>0$ such that

$$
\left|K_{t}(g)\right| \leq c t^{-D /(2 m)} e^{-b\left(\varrho_{A}(g)^{2 m} / t\right)^{1 /(2 m-1)}}
$$

for all $t \geq 1$ and $g \in G$. For $m=1$, it is well known that $H=H^{(1)}$ satisfies global Gaussian bounds (see, for example, [19]). If $G$ is nilpotent, or more generally, $G$ is a local direct product of a nilpotent group and a compact group, and $m$ is arbitrary, then $H$ satisfies global Gaussian bounds (see $[13,8]$, and earlier works $[10,3,15]$ dealing with homogeneous groups).

However, when $G$ is the universal cover of the solvable group of Euclidean motions of the plane, it was discovered in [12] that there are choices of $a_{1}, \ldots, a_{d^{\prime}}$ for which $H$ does not satisfy global Gaussian bounds when $m \geq 2$. For such choices one has $c^{-1} t^{-\mu} \leq\left\|K_{t}\right\|_{\infty} \leq c t^{-\mu}$ for all $t \geq 1$, with some $\mu>D /(2 m)$. Note that the author [6] recently established the upper bound $\left\|K_{t}\right\|_{\infty} \leq c t^{-D /(2 m)}$ for $t \geq 1$, for any $G$ and any list $a_{1}, \ldots, a_{d^{\prime}}$.

The aim of this paper is to give a general condition on $a_{1}, \ldots, a_{d^{\prime}}$ sufficient for global Gaussian bounds. To state it, suppose that $G$ is solvable, and let $\mathfrak{n} \subseteq \mathfrak{g}$ denote the nilradical (the largest nilpotent ideal) of $\mathfrak{g}$. To the solvable algebra $\mathfrak{g}=(\mathfrak{g},[\cdot, \cdot])$ we can associate its nilshadow $\mathfrak{g}_{N}$, which is a nilpotent Lie algebra with $\mathfrak{g}=\mathfrak{g}_{N}$ as vector spaces and Lie bracket $[\cdot, \cdot]_{N}$ (see, for example, $[1,2,9]$ ). Let $\mathfrak{g}_{N ; 1} \supseteq \mathfrak{g}_{N ; 2} \supseteq \cdots$ be the lower central series of $\mathfrak{g}_{N}$, defined by $\mathfrak{g}_{N ; 1}=\mathfrak{g}_{N}$ and $\mathfrak{g}_{N ; j+1}=\left[\mathfrak{g}_{N}, \mathfrak{g}_{N ; j}\right]_{N} \subseteq \mathfrak{g}_{N ; j}, j \in \mathbb{N}$. We say that the generating list $a_{1}, \ldots, a_{d^{\prime}}$ is nice of order $k$, where $k \in \mathbb{N}$, if there exists a Cartan subalgebra $\mathfrak{w}$ of $\mathfrak{g}$ such that each $a_{i}=v_{i}+y_{i}$, where $v_{i} \in \mathfrak{w}, y_{i} \in \mathfrak{n}$, and

$$
\left(\operatorname{ad} v_{i}\right)^{n} y_{i} \in \mathfrak{g}_{N ; n+1}
$$

for all $n \in\{1, \ldots, k-1\}$ and $i \in\left\{1, \ldots, d^{\prime}\right\}$. Here $(\operatorname{ad} x) y=[x, y], x, y \in \mathfrak{g}$, is the adjoint representation of $\mathfrak{g}$, and we recall that a Cartan subalgebra is a nilpotent subalgebra which equals its own normalizer.

Theorem 1.1. Assume that $G$ is solvable. If $a_{1}, \ldots, a_{d^{\prime}}$ is nice of order $m$, then $H=H^{(m)}$ satisfies global Gaussian bounds.

We conjecture that, for $G$ solvable and simply connected, niceness of order $m$ is necessary as well as sufficient for global Gaussian bounds for $H^{(m)}$.

Theorem 1.1 actually has an extension for any group $G$ of polynomial growth, which is more complicated to state: see Remark 2.6 below.

Theorem 1.1 will be derived from the following theorem of independent interest (and which is not restricted to the solvable case). Denote by $e$ the identity of $G$.

Theorem 1.2. Let $m \in \mathbb{N}$. Suppose there exist a family $\left(\eta_{R}\right)_{R \geq 1}$ of $C^{\infty}$-smooth functions on $G$ and a constant $c>0$ such that $0 \leq \eta_{R} \leq 1$, 
$\eta_{R}(e)=1$, the support of $\eta_{R}$ is contained in $B(c R)$, and

$$
\left\|A_{i}^{k} \eta_{R}\right\|_{\infty} \leq c R^{-k}
$$

for all $i \in\left\{1, \ldots, d^{\prime}\right\}, k \in\{1, \ldots, m\}$ and $R \geq 1$. Then $H=H^{(m)}$ satisfies global Gaussian bounds.

The above theorems are proved in Section 2. The effort of the proofs is reduced by utilizing work of the author [7] which shows that, to obtain Gaussian bounds for group-invariant semigroups, it suffices to verify certain $L^{2}$ "off-diagonal" estimates.

Let us notice some special cases and examples of Theorem 1.1. Every generating list is nice of order 1 . If $G$ is nilpotent, then $\mathfrak{g}=\mathfrak{g}_{N}=\mathfrak{n}$ as Lie algebras so that any generating list is nice of all orders. When $G$ is solvable, and $\mathfrak{w}$ is a Cartan subalgebra of $\mathfrak{g}$, any generating list satisfying $\left\{a_{1}, \ldots, a_{d^{\prime}}\right\} \subseteq \mathfrak{w} \cup \mathfrak{n}$ is nice of all orders. Since $\mathfrak{g}=\mathfrak{w}+\mathfrak{n}$ (see for example [9, pp. 64-65]) where the sum need not be direct, we see that nice generating lists certainly exist.

If $G$ is solvable and if $\mathfrak{g}_{N}$ is an abelian Lie algebra, then niceness of order $k, k \geq 2$, means that for some Cartan subalgebra $\mathfrak{w}, a_{i}=v_{i}+y_{i}$ with $v_{i} \in \mathfrak{w}, y_{i} \in \mathfrak{n}$, and $\left[v_{i}, y_{i}\right]=0$ for all $i$. Thus, for such $G$, the condition is independent of $k$ when $k \geq 2$. On the other hand, by considering solvable groups $G$ for which $\mathfrak{g}_{N}$ is non-abelian with large nilpotent rank, for any $k$ one can construct examples of generating lists which are nice of order $k$ but not of order $k+1$. We leave the details to the reader.

For the example of the group $G$ considered in [12], $\mathfrak{g}=\operatorname{span}\left\{b_{1}, b_{2}, b_{3}\right\}$ is a three-dimensional Lie algebra with non-zero commutation relations $\left[b_{1}, b_{2}\right]$ $=b_{3},\left[b_{1}, b_{3}\right]=-b_{2}$, and $\mathfrak{n}=\operatorname{span}\left\{b_{2}, b_{3}\right\}$. Let $a_{1}, a_{2}, a_{3}$ be a vector space basis of $\mathfrak{g}$. Since the Cartan subalgebras are just the one-dimensional subspaces of $\mathfrak{g}$ complementary to $\mathfrak{n}$, and $\mathfrak{g}_{N}$ is abelian, one sees that $a_{1}, a_{2}, a_{3}$ is nice of order $k(k \geq 2)$ if and only if exactly two of the $a_{i}$ are in $\mathfrak{n}$. The latter condition was noticed in [12].

Let us note a converse to Theorem 1.2.

THEOREM 1.3. Suppose that $H=H^{(m)}$ satisfies global Gaussian bounds. Then there exist smooth functions $\left(\eta_{R}\right)_{R \geq 1}$ on $G$ with properties as in the statement of Theorem 1.2.

To prove Theorem 1.3, choose a non-decreasing function $F \in C^{\infty}(\mathbb{R})$ with $F(x)=0$ for all $x \leq 2^{-1}$ and $F(x)=1$ for $x \geq 1$, and set

$$
\eta_{R}(g)=F\left(K_{R^{2 m}}(g) / K_{R^{2 m}}(e)\right), \quad g \in G .
$$

To verify the desired properties of $\eta_{R}$ is an easy adaption of arguments of [5, Section 2], and we omit further details.

Combining Theorems 1.2 and 1.3 gives the following interesting result. 
Corollary 1.4. Let $m_{1}, m_{2} \in \mathbb{N}$ with $m_{1}>m_{2}$. If $H^{\left(m_{1}\right)}$ satisfies global Gaussian bounds on $G$, then $H^{\left(m_{2}\right)}$ satisfies global Gaussian bounds on $G$.

Finally, let us speculate about heat kernel estimates for an arbitrary choice of $a_{1}, \ldots, a_{d^{\prime}}$. We conjecture that there exists a suitable function $\varrho_{A, m}: G \rightarrow[0, \infty)$, depending on $A_{1}, \ldots, A_{d^{\prime}}$ and on $m$, such that $\varrho_{A, m} \leq c \varrho_{A}$ and

$$
\left|K_{t}(g)\right| \leq c t^{-\widetilde{D} /(2 m)} e^{-b\left(\varrho_{A, m}(g)^{2 m} / t\right)^{1 /(2 m-1)}}, \quad\left\|K_{t}\right\|_{\infty} \geq c^{-1} t^{-\widetilde{D} /(2 m)}
$$

for all $t \geq 1$ and $g \in G$. Here, $\widetilde{D} \geq D$ denotes a global dimension associated with $\varrho_{A, m}$, that is, $d g\left(\left\{g \in G: \varrho_{A, m}(g)<r\right\}\right) \simeq r^{\widetilde{D}}$ for $r \geq 1$. The main difficulty in proving this conjecture is apparently to define a suitable $\varrho_{A, m}$; the results already stated suggest that $\varrho_{A, m}$ will be sensitive to perturbations of $A_{1}, \ldots, A_{d^{\prime}}$ or $m$.

\section{Proofs}

Proof of Theorem 1.2. In general, $c, c^{\prime}, b, \omega$ and so on denote positive constants whose value may change from line to line. We first construct functions $\varphi_{R}$ with similar properties to the given functions $\eta_{R}$, and with the symmetry property $\varphi_{R}\left(g^{-1}\right)=\varphi_{R}(g)$.

LEMMA 2.1. There exist $c>0$ and functions $\varphi_{R} \in C_{\mathrm{c}}^{\infty}(G), R \geq 1$, such that $0 \leq \varphi_{R} \leq 1, \varphi_{R}\left(g^{-1}\right)=\varphi_{R}(g)$ for all $g \in G, \varphi_{R}$ is supported in $B(c R)$, $\varphi_{R}(g)=1$ whenever $g \in B\left(c^{-1} R\right)$, and

$$
\left\|A_{i}^{k} \varphi_{R}\right\|_{\infty} \leq c R^{-k}
$$

for all $R \geq 1, i \in\left\{1, \ldots, d^{\prime}\right\}$ and $k \in\{1, \ldots, m\}$.

Proof. For $R \geq 1$ let $t_{R}=\int_{G} \eta_{R}^{2}$ and $\widetilde{\eta}_{R}(g)=\eta_{R}\left(g^{-1}\right)$, and define

$$
\widehat{\eta}_{R}:=t_{R}^{-1}\left(\eta_{R} * \widetilde{\eta}_{R}\right)
$$

where $*$ denotes convolution of functions on $G$. So $A_{i}^{k} \widehat{\eta}_{R}=t_{R}^{-1}\left(A_{i}^{k} \eta_{R}\right) * \widetilde{\eta}_{R}$, and there is an estimate $c^{-1} R^{D} \leq t_{R} \leq c R^{D}$ for all $R \geq 1$. It is then straightforward to check that the $\widehat{\eta}_{R}$ have the same properties as $\eta_{R}$, and moreover $\widehat{\eta}_{R}\left(g^{-1}\right)=\widehat{\eta}_{R}(g), g \in G$. Since $\left\|A_{i} \widehat{\eta}_{R}\right\|_{\infty} \leq c R^{-1}$ and $\widehat{\eta}_{R}(e)=1$, one easily shows that there is $c^{\prime}>0$ with

$$
\widehat{\eta}_{R}(g) \geq 2^{-1}
$$

for all $g \in B\left(\left(c^{\prime}\right)^{-1} R\right)$ and $R \geq 1$. Choose a $C^{\infty}$-smooth function $F:[0, \infty) \rightarrow$ $[0,1]$ such that $F(0)=0$ and $F(x)=1$ for all $x \geq 2^{-1}$. The functions $\varphi_{R}:=F \circ \widehat{\eta}_{R}$ have the required properties.

Borrowing an idea of [14], we define a function $\varrho: G \rightarrow[1, \infty)$ by

$$
\varrho(g)=1+\sum_{j=1}^{\infty}\left(1-\varphi_{j}(g)\right) .
$$


From the properties of $\varphi_{R}$, one sees that the sum is locally finite, so $\varrho$ is a smooth function, and that

$$
c^{-1}\left(1+\varrho_{A}\right) \leq \varrho \leq c\left(1+\varrho_{A}\right) .
$$

Thus $\varrho$ is a smooth approximation of $\varrho_{A}$. Moreover, $\varrho(g)=\varrho\left(g^{-1}\right)$ for all $g \in G$. For $g \in G$ with $\varrho_{A}(g)>1$, the properties of $\varphi_{R}$ yield

$$
\left|\left(A_{i}^{k} \varrho\right)(g)\right| \leq \sum_{c^{-1} \varrho_{A}(g) \leq j \leq c \varrho_{A}(g)}\left\|A_{i}^{k} \varphi_{j}\right\|_{\infty} \leq c^{\prime} \varrho_{A}(g)^{1-k}
$$

for all $k \in\{1, \ldots, m\}$. Hence

$$
\left|\left(A_{i}^{k} \varrho\right)(g)\right| \leq c^{\prime \prime} \varrho(g)^{1-k}
$$

for all $g \in G$ and $k \in\{1, \ldots, m\}$. Taking $k=1$ in (5), we deduce that

$$
|\varrho(h)-\varrho(g h)| \leq c \varrho A(g) \sum_{i=1}^{d^{\prime}}\left\|A_{i} \varrho\right\|_{\infty} \leq c^{\prime} \varrho(g)
$$

for all $g, h \in G$, and consequently $\varrho(g h) \leq c(\varrho(g)+\varrho(h))$ for all $g, h \in G$.

In what follows, write $M=2 m$, denote by $U_{\lambda}$ the multiplication operator $f \mapsto e^{\lambda \varrho} f$ for $\lambda \in \mathbb{R}$, and let $L=L_{G}$ be the left regular representation of $G$ with $(L(g) f)(h)=f\left(g^{-1} h\right), g, h \in G$, for functions $f: G \rightarrow \mathbb{C}$. We will establish estimates

$$
\begin{gathered}
\left\|U_{\lambda} S_{t} U_{-\lambda}\right\|_{2 \rightarrow 2} \leq c e^{\omega \lambda^{M} t} \\
\left\|(I-L(g)) U_{\lambda} S_{t} U_{-\lambda}\right\|_{2 \rightarrow 2} \leq c \varrho(g) t^{-1 / M} e^{\omega \lambda^{M} t}
\end{gathered}
$$

for all $t \geq 1, \lambda \in \mathbb{R}$ and $g \in G$ such that $\varrho(g) \leq t^{1 / M}$ (where $\|\cdot\|_{2 \rightarrow 2}$ denotes the operator norm for bounded operators in $L^{2}$ ). Then it follows from Theorem 2.3 and Remark 2.4 of [7], together with the local estimate (1), that

$$
\left|K_{n}(g)\right| \leq c n^{-D / M} \exp \left(-b\left(\varrho(g)^{M} / n\right)^{1 /(M-1)}\right)
$$

for all $n \in \mathbb{N}$ and $g \in G$. In this inequality, we may replace $\varrho$ with $\varrho_{A}$, because of (4). Then using the semigroup property $K_{t}=K_{t-n} * K_{n}$ and (1), we easily deduce global Gaussian bounds for $H$.

Therefore, to complete the proof of Theorem 1.2 it remains to verify (6).

The proof of (6) is an adaption of the usual Davies perturbation technique. Introduce the set $\mathcal{E}_{m}$ of smooth functions $\psi: G \rightarrow \mathbb{R}$ such that $\left\|A_{i}^{k}\right\|_{\infty}<\infty$ for all $i \in\left\{1, \ldots, d^{\prime}\right\}$ and $k \in\{1, \ldots, m\}$; we do not assume that $\psi$ is bounded. Because of the relation

$$
e^{\psi} A_{i}\left(e^{-\psi} f\right)=A_{i} f-\left(A_{i} \psi\right) f
$$

we may consider $H_{\psi}:=e^{\psi} H e^{-\psi}$ as a differential operator which is a perturbation of $H$ by terms of order less than $2 m$ with bounded coefficients. 
Define a functional $\sigma_{m}$ on $\mathcal{E}_{m}$ by

$$
\sigma_{m}(\psi)=\sum_{i=1}^{d^{\prime}} \sum_{k=1}^{m}\left(\left\|A_{i}^{k} \psi\right\|_{\infty}\right)^{1 / k}
$$

we usually abbreviate $\sigma_{m}=\sigma$. In the next two lemmas, we will see that this functional seems well adapted to the study of off-diagonal estimates for $H$. Notice that the lemmas apply to an arbitrary generating list $a_{1}, \ldots, a_{d^{\prime}}$, that is, they make no use of the hypothesis of Theorem 1.2.

LEMma 2.2. There exists $c>0$ such that

$$
\left|(H f, f)-\left(H_{\psi} f, f\right)\right| \leq 2^{-1}(H f, f)+c \sigma(\psi)^{M}\|f\|_{2}^{2}
$$

for all $f \in C_{\mathrm{c}}^{\infty}(G)$ and $\psi \in \mathcal{E}_{m}$. Consequently, there is $c_{1}>0$ with

$$
\operatorname{Re}\left(H_{\psi} f, f\right) \geq-c_{1} \sigma(\psi)^{M}\|f\|_{2}^{2}
$$

for all $f \in C_{\mathrm{c}}^{\infty}$ and $\psi \in \mathcal{E}_{m}$.

Proof. The second estimate of the lemma follows easily from the first. Let us sketch the proof of the first estimate, which is a variation of standard arguments (see [4] and [5, pp. 58-59]). Write

$$
(H f, f)-\left(H_{\psi} f, f\right)=\sum_{i=1}^{d^{\prime}}\left(\left(A_{i}^{m} f, A_{i}^{m} f\right)-\left(e^{\psi} A_{i}^{m} e^{-\psi} f, e^{-\psi} A_{i}^{m} e^{\psi} f\right)\right)
$$

and apply (7) to expand the left side as a sum of terms each of the form

$$
T(f)=c\left(\left(A_{i}^{n_{1}} \psi\right) \cdots\left(A_{i}^{n_{p}} \psi\right) A_{i}^{k_{1}} f, A_{i}^{k_{2}} f\right),
$$

where $p \in \mathbb{N}, n_{1}, \ldots, n_{p} \in\{1, \ldots, m\}, k_{1}, k_{2} \in\{0,1, \ldots, m\}$, and $n_{1}+\cdots+$ $n_{p}+k_{1}+k_{2}=2 m=M$. Now set $r=n_{1}+\cdots+n_{p} \in\{1, \ldots, M\}$, and apply a standard interpolation inequality

$$
\left\|A_{i}^{k} f\right\|_{2} \leq c\left\|A_{i}^{m} f\right\|_{2}^{k / m}\|f\|_{2}^{1-(k / m)} \leq c(H f, f)^{k / M}\|f\|_{2}^{1-(k / m)},
$$

which is valid for $k \in\{0,1, \ldots, m\}$. We obtain

$$
\begin{aligned}
|T(f)| & \leq c \sigma(\psi)^{r}\left\|A_{i}^{k_{1}} f\right\|_{2}\left\|A_{i}^{k_{2}} f\right\|_{2} \leq c^{\prime}(H f, f)^{1-(r / M)}\left(\sigma(\psi)^{M}\|f\|_{2}^{2}\right)^{r / M} \\
& \leq \varepsilon(H f, f)+c \varepsilon^{-(M-r) / r} \sigma(\psi)^{M}\|f\|_{2}^{2}
\end{aligned}
$$

for all $\varepsilon>0$, and the lemma follows.

The next lemma is deduced from Lemma 2.2 by a standard line of reasoning, and we omit the details (see [5, pp. 55-56] or [4]).

LEMma 2.3. The operator $H_{\psi}$ generates a semigroup $S_{t}^{\psi}=e^{\psi} S_{t} e^{-\psi}$ in $L^{2}$, satisfying the estimates

$$
\left\|S_{t}^{\psi}\right\|_{2 \rightarrow 2}+t\left\|H_{\psi} S_{t}^{\psi}\right\|_{2 \rightarrow 2} \leq c e^{\omega \sigma(\psi)^{M} t}, \quad\left\|A_{i}^{k} S_{t}^{\psi}\right\|_{2 \rightarrow 2} \leq c t^{-k / M} e^{\omega \sigma(\psi)^{M} t}
$$

for all $t>0, \psi \in \mathcal{E}_{m}, i \in\left\{1, \ldots, d^{\prime}\right\}$ and $k \in\{1, \ldots, m\}$. 
To prove (6) we consider separately the two cases $|\lambda| \geq 2^{-1}$ and $|\lambda|<2^{-1}$. In the first case, observe from (5) that $\lambda \varrho \in \mathcal{E}_{m}$ with an estimate

$$
\sigma(\lambda \varrho) \leq c \sum_{i=1}^{d^{\prime}} \sum_{k=1}^{m}|\lambda|^{1 / k} \leq c^{\prime}|\lambda| .
$$

Therefore, from Lemma 2.3 and the standard inequality (see [18, p. 268])

$$
\|(I-L(g)) f\|_{2} \leq \varrho_{A}(g)\left(\sum_{i=1}^{d^{\prime}}\left\|A_{i} f\right\|_{2}^{2}\right)^{1 / 2}
$$

we obtain (6) for $|\lambda| \geq 2^{-1}$.

To deal with the second case, define $\varrho_{j}=1+\sum_{s=j}^{\infty}\left(1-\varphi_{s}\right)$ for each $j \in \mathbb{N}$. Then $\varrho_{1}=\varrho$ and

$$
\left\|\varrho-\varrho_{j}\right\|_{\infty} \leq j-1, \quad\left\|A_{i}^{k} \varrho_{j}\right\|_{\infty} \leq c j^{1-k}
$$

for all $j \in \mathbb{N}$ and $k \in\{1, \ldots, m\}$, where the second inequality is proved in a similar way to (5). Given $0<|\lambda|<2^{-1}$, let $j$ be the greatest integer less than or equal to $|\lambda|^{-1}$. Then $2^{-1} \leq|\lambda| j \leq 1, \sigma\left(\lambda \varrho_{j}\right) \leq c|\lambda|$, and from Lemma 2.3 we get

$$
\begin{aligned}
\left\|e^{\lambda \varrho} S_{t} e^{-\lambda \varrho}\right\|_{2 \rightarrow 2} & \leq\left\|e^{\lambda\left(\varrho-\varrho_{j}\right)}\right\|_{2 \rightarrow 2}\left\|e^{\lambda \varrho_{j}} S_{t} e^{-\lambda \varrho_{j}}\right\|_{2 \rightarrow 2}\left\|e^{-\lambda\left(\varrho-\varrho_{j}\right)}\right\|_{2 \rightarrow 2} \\
& \leq c^{\prime} e^{\omega \lambda^{M} t},
\end{aligned}
$$

where $c^{\prime}, \omega$ are constants independent of $\lambda$. This yields the first estimate of (6) for $|\lambda|<2^{-1}$ (the case $\lambda=0$ is trivial). The second estimate of (6) may be proved in a similar way by estimating the operators

$$
A_{i} e^{\lambda \varrho} S_{t} e^{-\lambda \varrho}=\left(A_{i}\left(e^{\lambda\left(\varrho-\varrho_{j}\right)}\right)\right) S_{t}^{\lambda \varrho_{j}} e^{-\lambda\left(\varrho-\varrho_{j}\right)}+e^{\lambda\left(\varrho-\varrho_{j}\right)} A_{i} S_{t}^{\lambda \varrho_{j}} e^{-\lambda\left(\varrho-\varrho_{j}\right)}
$$

for $0<|\lambda|<2^{-1}$, and applying (9). The proof of Theorem 1.2 is complete.

Proof of Theorem 1.1. Let $G$ be solvable and fix a Cartan subalgebra $\mathfrak{w}$ of $\mathfrak{g}$ such that $a_{i}=v_{i}+y_{i}, v_{i} \in \mathfrak{w}, y_{i} \in \mathfrak{n}$, and such that (3) holds for all $n \in\{1, \ldots, m-1\}$. From $\mathfrak{w}$ one may construct the nilshadow Lie group $G_{N}=G_{N}(\mathfrak{w})$, with Lie algebra $\mathfrak{g}_{N}$, such that $G=G_{N}$ are identified as manifolds and $\mathfrak{g}=\mathfrak{g}_{N}$ are identified as vector spaces. For details see $[9,1,2]$ : in these references $G_{N}$ is constructed starting from a subspace $\mathfrak{v} \subseteq \mathfrak{w}$ satisfying $\mathfrak{g}=\mathfrak{v} \oplus \mathfrak{n}$, but the construction is actually independent of the choice of $\mathfrak{v}$ within $\mathfrak{w}$ (see [9, pp. 78-80]). For $x \in \mathfrak{g}$, we will write

$$
X=d L_{G}(x), \quad \tilde{X}=d L_{G_{N}}(x)
$$

respectively for the $G$-right invariant vector field and the $G_{N}$-right invariant vector field corresponding to $x$. In particular, $A_{i}=V_{i}+Y_{i}$, where $V_{i}=$ $d L_{G}\left(v_{i}\right), Y_{i}=d L_{G}\left(y_{i}\right)$. The following lemma is contained in the analysis of $[2,9]$. 
LEMMA 2.4. One has

$$
(X f)(g)=\left(d L_{G}(x) f\right)(g)=\left(\left(d L_{G_{N}}\left(\bar{T}\left(g^{-1}\right) x\right)\right) f\right)(g)
$$

for all $x \in \mathfrak{g}, f \in C_{\mathrm{c}}^{\infty}(G)$ and $g \in G$. Here $\bar{T}$ is a certain representation of $G$ in the vector space $\mathfrak{g}$, such that $\bar{T}(g) x=x$ for all $x \in \mathfrak{w}$ and $g \in G$, and $\bar{T}(\exp y)=I$ for all $y \in \mathfrak{n}$. Moreover, the $\bar{T}(g)$ are orthogonal transformations with respect to a suitably chosen inner product on $\mathfrak{g}$, and $\bar{T}(g)(\mathfrak{n}) \subseteq \mathfrak{n}$, $\bar{T}(g)\left(\mathfrak{g}_{N ; j}\right) \subseteq \mathfrak{g}_{N ; j}$ for all $j$.

LEMma 2.5. There exists a family $\left(\eta_{R}\right)_{R \geq 1}$ of smooth functions on $G_{N}$ such that $0 \leq \eta_{R} \leq 1, \eta_{R}(e)=1$, and $\eta_{R}$ is supported in $B(c R)$. Moreover, for any $k \in \mathbb{N}$ and $z_{1}, \ldots, z_{k} \in \mathfrak{g}$ with $z_{j} \in \mathfrak{g}_{N ; m_{j}}$ for some $m_{1}, \ldots, m_{k} \in \mathbb{N}$, there is $c^{\prime}>0$ such that

$$
\left\|\widetilde{Z}_{1} \cdots \widetilde{Z}_{k} \eta_{R}\right\|_{\infty} \leq c^{\prime} R^{-\left(m_{1}+\cdots+m_{k}\right)}
$$

for all $R \geq 1$, where $\widetilde{Z}_{j}=d L_{G_{N}}\left(z_{j}\right)$.

Proof. The distances on $G$ and $G_{N}$ are equivalent at infinity, that is, if $\widetilde{\varrho}$ denotes a Carathéodory modulus on $G_{N}$ then one has an estimate $c^{-1}(1+\widetilde{\varrho}) \leq 1+\varrho_{A} \leq c(1+\widetilde{\varrho})$ (see [9] or [2]). Then the lemma follows from results of [14] and the nilpotency of $G_{N}$.

Alternatively, one can prove the lemma more directly by fixing a suitable $\eta_{1} \in C_{\mathrm{c}}^{\infty}\left(G_{N}\right)$ and setting $\eta_{R}(g)=\eta_{1}\left(\tau_{R^{-1}}(g)\right)$ for $R>1$, where $\tau_{\delta}, \delta>0$, are dilations "at infinity" on the nilpotent group $G_{N}$ (see, for example, [1, Section 5] or [17] for these dilations). We skip the details.

Set $w_{i, n}=\left(\operatorname{ad} v_{i}\right)^{n} y_{i}$ and $W_{i, n}=d L_{G}\left(w_{i, n}\right)$ for $n \in \mathbb{N}_{0}=\{0,1, \ldots\}$. Expanding $A_{i}^{m}=\left(V_{i}+Y_{i}\right)^{m}$ and reordering terms yields an expression

$$
A_{i}^{m}=V_{i}^{m}+Y_{i}^{m}+\sum c_{l_{0}, \ldots, l_{s}} W_{i, l_{1}} \ldots W_{i, l_{s}} V_{i}^{l_{0}}
$$

where the sum is over all $s \geq 1$ and $l_{0}, l_{1}, \ldots, l_{s} \in\{0,1, \ldots, m-1\}$ with $l_{0}+l_{1}+\cdots+l_{s}+s=m$ and where $c_{l_{0}, \ldots, l_{s}}$ are constants.

Since $v_{i} \in \mathfrak{w}$, it follows from Lemma 2.4 that $V_{i}=d L_{G_{N}}\left(v_{i}\right)$, that is, $V_{i}$ is a $G_{N}$-invariant vector field. Also, given $n \in\{0,1, \ldots, m-1\}$ and $i \in\left\{1, \ldots, d^{\prime}\right\}$, since $w_{i, n} \in \mathfrak{n} \cap \mathfrak{g}_{N ; n+1}$ we can use Lemma 2.4 to express

$$
W_{i, n}=\sum_{j} \xi_{j} \widetilde{Z}_{j}
$$

for some fields $\widetilde{Z}_{j}=d L_{G_{N}}\left(z_{j}\right)$ with $z_{j} \in \mathfrak{n} \cap \mathfrak{g}_{N ; n+1}$. The $\xi_{j}: G \rightarrow \mathbb{R}$ are smooth bounded functions which are constant in the $\mathfrak{n}$-directions, that is, $\widetilde{Y} \xi_{j}=0$ for any $y \in \mathfrak{n}$ (for further details, see for example [9, p. 183]).

By combining these observations with (11), we may express $A_{i}^{m}$ as a sum of terms each of the form $\xi \widetilde{Z}_{1} \ldots \widetilde{Z}_{k}$, where $\xi$ are smooth bounded functions and $z_{j} \in \mathfrak{g}_{N ; m_{j}}$ with $m_{1}+\cdots+m_{k} \geq m$. Therefore, by Lemma 2.5 one has $\left\|A_{i}^{m} \eta_{R}\right\|_{\infty} \leq c R^{-m}$ for $R \geq 1$. Since $\left\|\eta_{R}\right\|_{\infty}=1$, by interpolation we get 
$\left\|A_{i}^{k} \eta_{R}\right\|_{\infty} \leq c^{\prime} R^{-k}$ for all $k \in\{1, \ldots, m\}$. Theorem 1.1 now follows from Theorem 1.2.

REMARK 2.6. Let us note a generalization of Theorem 1.1 for any Lie group $G$ of polynomial growth. In this general case, $\mathfrak{g} \supseteq \mathfrak{q} \supseteq \mathfrak{n}$ where $\mathfrak{q}$ is the solvable radical and $\mathfrak{n}$ is the nilradical of $\mathfrak{g}$. A subalgebra $\mathfrak{w} \subseteq \mathfrak{g}$ will be called a generalized Cartan subalgebra of $\mathfrak{g}$ if $\mathfrak{w}=\mathfrak{m} \oplus \mathfrak{w}_{0}$ where $\mathfrak{m}$ is a Levi subalgebra of $\mathfrak{g}$ (that is, $\mathfrak{m}$ is a semisimple subalgebra with $\mathfrak{g}=\mathfrak{m} \oplus \mathfrak{q}$ ) and $\mathfrak{w}_{0} \subseteq \mathfrak{q}$ is a Cartan subalgebra of the algebra $\mathfrak{q}_{0}(\mathfrak{m}):=\{x \in \mathfrak{q}:[\mathfrak{m}, x]=\{0\}\}$ A generalized Cartan subalgebra satisfies $\mathfrak{g}=\mathfrak{w}+\mathfrak{n}$ (see, for instance, $\left[9\right.$, pp. 64-65]). Let $\mathfrak{q}_{N ; 1} \supseteq \mathfrak{q}_{N ; 2} \supseteq \cdots$ be the lower central series of the nilshadow $\mathfrak{q}_{N}$ associated with $\mathfrak{q}$. Then we have:

THEOREM 2.7. Let $a_{1}, \ldots, a_{d^{\prime}}$ be a generating list such that there exists a generalized Cartan subalgebra $\mathfrak{w}$ with $a_{i}=v_{i}+y_{i}, v_{i} \in \mathfrak{w}, y_{i} \in \mathfrak{n}$, and $\left(\operatorname{ad} v_{i}\right)^{n} y_{i} \in \mathfrak{q}_{N ; n+1}$ for all $n \in\{1, \ldots, m-1\}$ and all $i$. Then $H=H^{(m)}$ satisfies global Gaussian bounds.

The proof is a straightforward extension of the above proof of Theorem 1.1, since one knows suitable generalizations of Lemmas 2.4 and 2.5 (see again $[9,2]$ for the structure theory of $G$ ). We omit the details.

Acknowledgements. This work was carried out with financial support from the Australian Research Council (ARC) Centre of Excellence for Mathematics and Statistics of Complex Systems (MASCOS). I thank Derek Robinson and Tom ter Elst for helpful discussions.

\section{References}

[1] G. Alexopoulos, An application of homogenization theory to harmonic analysis: Harnack inequalities and Riesz transforms on Lie groups of polynomial growth, Canad. J. Math. 44 (1992), 691-727.

[2] -, Sub-Laplacians with drift on Lie groups of polynomial volume growth, Mem. Amer. Math. Soc. 155 (2002), no. 739.

[3] P. Auscher, A. F. M. ter Elst and D. W. Robinson, On positive Rockland operators, Colloq. Math. 67 (1994), 197-216.

[4] E. B. Davies, Uniformly elliptic operators with measurable coefficients, J. Funct. Anal. 132 (1995), 141-169.

[5] N. Dungey, Higher order operators and Gaussian bounds on Lie groups of polynomial growth, J. Operator Theory 46 (2001), 45-61.

[6] - Some conditions for decay of convolution powers and heat kernels on groups, Canad. J. Math., to appear.

[7] -, On Gaussian kernel estimates on groups, Colloq. Math. 100 (2004), 77-90.

[8] N. Dungey, A. F. M. ter Elst and D. W. Robinson, Asymptotics of sums of subcoercive operators, ibid. 82 (1999), 231-260.

[9] - - - - - Analysis on Lie Groups with Polynomial Growth, Progr. Math. 214, Birkhäuser, Boston, 2003. 
[10] J. Dziubański, W. Hebisch and J. Zienkiewicz, Note on semigroups generated by positive Rockland operators on graded homogeneous groups, Studia Math. 110 (1994), $115-126$.

[11] A. F. M. ter Elst and D. W. Robinson, Weighted subcoercive operators on Lie groups, J. Funct. Anal. 157 (1998), 88-163.

[12] - - - On anomalous asymptotics of heat kernels, in: Evolution Equations and Their Applications to Physical and Life Sciences, G. Lumer and L. Weis (eds.), Marcel Dekker, New York, 2001, 89-103.

[13] A. F. M. ter Elst, D. W. Robinson and A. Sikora, Heat kernels and Riesz transforms on nilpotent Lie groups, Colloq. Math. 74 (1997), 191-218.

[14] - - - -, Riesz transforms and Lie groups of polynomial growth, J. Funct. Anal. 162 (1999), 14-51.

[15] W. Hebisch, Sharp pointwise estimate for the kernels of the semigroup generated by sums of even powers of vector fields on homogeneous groups, Studia Math. 95 (1989), 93-106.

[16] - Estimates on the semigroups generated by left invariant operators on Lie groups, J. Reine Angew. Math. 423 (1992), 1-45.

[17] A. Nagel, F. Ricci and E. M. Stein, Harmonic analysis and fundamental solutions on nilpotent Lie groups, in: Analysis and Partial Differential Equations, C. Sadosky (ed.), Lecture Notes in Pure and Appl. Math. 122, Marcel Dekker, New York, 1990, 249-275.

[18] D. W. Robinson, Elliptic Operators and Lie Groups, Oxford Math. Monogr., Oxford Univ. Press, Oxford, 1991.

[19] N. T. Varopoulos, L. Saloff-Coste and T. Coulhon, Analysis and Geometry on Groups, Cambridge Tracts in Math. 100, Cambridge Univ. Press, Cambridge, 1992.

School of Mathematics

University of New South Wales

Sydney 2052, Australia

E-mail: dungey@maths.unsw.edu.au 